\title{
Representación del conocimiento y clasificación en el ámbito bibliotecológico
}

\author{
Ramiro Lafuente López*
}

\section{RESUMEN}

Losservi ciosbibliotecarios hanes ta do orien ta dos al al macenamien to yrecuperación de la información. Sin embargo, con la introducción de la tecnología de los sistemas expertos, se ha mostrado una tendencia a que la idea de la búsqueda de in for ma ción bi blio gráfi ca no se de ten ga sim ple men te en la re cu pera ción del dato y la información relevante sino que se muestren las relaciones que existen entre diversosfenómenos que se en cuen tran re pre sen ta dos. De tal ma ne ra que no se tra taría de in da gar acer ca de un dato en sí, sino acer ca de la re la ción que un fe nó me no guar da con otro fe nó me no. En este ar tí cu lo se pre sen ta un es bo zo de lo que po dría ser el con tem plar la ca ta lo ga ción como un proble ma en don de en tran en jue go "objetos" y "relaciones entre objetos".

\section{ABSTRACT}

Library services have been oriented around the storage and recovery of information. nevertheless, with the introduction of technology of sophisticated systems, the re has been a growing ten dency affir minf that the idea of the search for bi blio graphi cal in for ma tion should not end sim ply with the re co very of the re le vant data and information but rather should demostrate the relations that exist between diverse phe no me na that are afound re pre sen ted. The re fo re, the search is not to find data in itself, but rather to dis co ver the re la tion that one phe no me non main tains with another. In this article an outline is sketched that contemplates cataloging as a problem in which "objects" and relationship' between objects" both play a part.

\section{LA BIBLIOTECOLOGIA EN LA ERA DEL SIMBOLO Y LA INFORMACION}

T a biblioteca destina su organización y _personal al propósito de cumplir fines decaráctercolectivoy de naturalezapública. La presencia de la Biblioteca adquiere sentidoúnicamenteenel contextodenecesida desy con duc ta de los miem bros deuna comunidad, lo cual significa que adquiere sentido y presencia en la comunidad a través de sus servicios. Cada comunidad entiende a la Biblioteca de un modo particular, y la dota de jui cios o va lo res, que le dan razón de ser y le atribuyen determinados significados.

A par tir del si gloXVIII la Bi blio te ca sedesen volvió en un con tex to so cial de servicio público que atendió a la idea de crear colecciones estables, con la intención de fortalecer la acumulación social del conocimiento por medio del contexto impreso.

La Biblioteca es una institución que forma partedelosfenómenosrelativosalproceso decomunicaciónhumanadelconocimientoy lain for ma ción, yad quie re sen ti doy razón de ser en re la ción a fe nó me nos comola Industria Editorial, la Educación, y la Instrucción Pública.

En el transcurso del siglo XIX el quehacer de la Biblioteca se orientó fundamentalmentealaorganiza ción de coleccionesdocumentales: a) Representativas de la cultura nacional (Bibliotecas Nacionales),

b) Deapoyoala investiga ciónyalaeducación (Bibliotecas Universitarias y Escolares),

c) Destinadas a instrucción pública, y a la difusióndelconocimientoeinformación deinteréspúblico(BibliotecasPúblicas).

La formación de estas colecciones atendió alafinalidad de crearunespaciodestinado al prés ta mo de las mis mas, a la vez que garantizó el acceso irrestricto a la cultura impre sa en todo mo men to, pues to que la colección de una biblioteca se establece con 
la idea de institucionalizar los servicios de présta mo de la colec ción, a fin de con ver tirlos en una actividad permanente.

Para facilitar la formación y préstamo de colecciones se crearon y desarrollaron sistemas dirigidos a servir de instrumento parare crearespacioen loscuales disponer una colec ción en for ma or de na day con los suficientes medios catalográficos que la describieran, con objeto de dara co nocerel tipo de do cu men to y sus con te ni dos te máticos. Almis motiem po que se idea ron sistemas para la formación y organización de colecciones, se generaron técnicas y sistemas para la difusiónsiste matiza dadelainformación relativa a los libros que se encontraban disponibles para su venta (Catálogosdeeditoresylibreros), delibros publicadossobredeterminadostemas (Bibliografías), y de los libros que formaban parte delas coleccio nes bibliote carias (Catálogos colectivos).

Alprin cipiodelsiglo XX la for ma ción de catálogos y bibliografías se agrupó conceptualmente bajo la denominación de control bibliográfico $e$ incluso se institucionalizaronactividadescomola forma ción de una Biblio grafía Univer sal y Catálogos colectivos regionales. Respecto al control bibliográfico podemos distinguir dosfenómenos: el con trolbibliográficoimpulsadopororganismosnacionalesointernacio nales, queatien de afinalidadesde investigación científica o de preservación de la cul tura; y el con trol bi blio gráfi co que surge como par te de la in dus tria edi to rial y de la información, el cual se traduce en índices, publicados en diversas formas tanto manualescomoau to matizadas, conunvalor comercial y sujetos a las condiciones propias del mercado.

Laidea del con trolbibliográficoimpulsada tanto por actividades institucionales de índole nacionale in ternacio nal, comoporlos productosbibliográficos delaindustriaedi torialy delain forma ción, se exten dió hasta ad qui rir unva lor so cial que le atribu ye una importancia fundamental en el proceso de comunicación y difusión del conocimiento y la in for ma ción, de esta ma ne ra a me diados del siglo XX, el control bibliográfico se transformó en un fenómeno al cual se subordinaronlosserviciosbibliotecarios. Esta idea, fue unfactorde cisivoen laso cialización delconocimientoacercadeladescripción bi bliográfica, lo que propició que a partir de mediadosdelsiglo XX, fue se co mún el uso de las referen ciasbibliográficascomopartede la elaboración de textos. La utilización generalizada de la referencia bibliográfica se convirtió en un mecanismo social de controlbibliográfico, gracias al cual es factible contar con un medio muy eficiente de búsqueda bibliográfica.
En la segunda década del siglo XX se crearon conocimientos, técnicas y sistemas para la formación, organización y préstamo de colecciones, dan do al pú bli co el acce so di rec to a los li bros por me dio de la es tantería abierta. Este sistema de préstamo, per mite arre glary re la cio nar los do cumentos que for man una colec ción, en tal for ma, que es factibleconvertirelespaciodestinado al aco mo do de una co lec ción, en un ins trumentodebúsque dadocumental conun grado de efi cien cia su pe rioral de cual quier instrumento catalográfico, y que puede alcanzar tanto cuantitativa como cualitativamente rendimientos crecientes en eficiencia, cuando se relaciona la estantería abierta con el uso de catálogos.

Para me dia dos del si gloXX los ser vi cios bi bliotecariosincluían la formación y organización de colecciones, así como su préstamo, yafue raporacceso directodelpúblico, como en el caso de la estantería abierta, o valién dose del ca tálogo, siel público no tiene un acceso directo a la colección. Además, losserviciosbibliotecarios, participanenel control bibliográficoatravésdelaformación de catálogos colectivos y la publicación de boletines de difusión de información bibliográfica acer ca de los do cu men tos que for man una colección. También, habíanincorporadoel servicio de consulta como un medio de conferir, tratar y discutir con el público acercadelaformadesatisfacersusrequerimientoen cuantoabúsque dabibliográfica ylasformas más ade cua daspara en con trar determinados conocimientos o información.

La idea del con trol bi blio grá fi coy la utilización de la automatización para el proceso dedatosbibliográficos, propicióla creación del concepto de servicio de información, establecien do un con tex to so cialque trans formó los servicios bibliotecarios al orientarlos fundamentalmente hacia el logro de la efi cien cia en el almacenamiento-recupera cióndeinformaciónbibliográfica. El con cep to de servicio de información se sustenta en las cre en cias, mi tos y va lo res que ro dean a la in for ma ción a par tir de me dia dos de este siglo, y alude a términos que tienen como cen tro de grave dad la in ten ción de proveer al usuario de información "relevante", en forma "rápida y oportuna", a partir del diagnóstico de sus "necesidades de información".

Losservicios de informa ción fun damen tal menteseencaminaron hacialasolu ción de problemasrelativosalcontrolbibliográfico, su conceptualización lo caracteriza como un ele men to esen cial para la difu sión y bús queda de información bibliográfica, y se encuentravinculadoafenómenosrelativos a la denominada Industria de la Información, la cual como fenómeno social representaungradomás deevolu cióntecnológicaenlossistemas de co mercialización dela
Industria Editorial. Los servicios de informa ción preten derau xiliaralosusua riosen labúsque da de in forma ción, porme dio de una variada parafernaliade sistemas automatizadosderecuperacióndeinformación bibliográfica, sujetostodosaundenominador común: un alto grado de eficiencia en la recuperación de datos (recuperación de información relevante), y herramientas anexas para la selección y descriminación de datos (selec ción dela in for ma ción pertinente), que en la práctica dan re sul ta dos de una probreza inaudita.

La ne cesidad de ha cer fun cio nala labiblioteca con du jo en la se gun da mi tad del si glo $\mathrm{XX}$ a la idea de lo grar una ma yor efi cien cia y rentabilidad en la operación de los servicios bibliotecarios substituyendo el describiry clasificarlacolección de unabiblioteca conformealasfinalidadesquesepersigan, por la compra de fichas estándar que proporcionan los servicios de información bibliográfica, lo cual reduce la actividad bibliote cariaa un pragma tismoque orillaala biblioteca a convertirse en esclava de la vaga idea de satisfacer las necesidades de información de los usuarios, alejando la posibilidadde vis lum brarque servi ciospodrían ser significa tivos en cuan to al de sempe ño de labiblio te ca en re la ción a las cos tumbres y necesidades de los miembros de una comunidad.

Los servicios bibliotecarios evidentemente se encuentran vinculados a la función histórica que ha desempeñado la Biblioteca en su pa pel de Ins ti tu ción de Cul tu ra orientada a la selección de materiales para formar colecciones documentales que den a entender o permitan conocer los contenidos de los documentos. Los servicios bibliotecariosformanpartedelosfenómenos pro pios de las for mas en como se ge ne ray transmiteelconocimien toylainformación en nuestra sociedad, y su implementación ydesarrollonecesariamentenosplanteainterrogantesacerca de la rela ción que existe odebe exis tiren tre las finalida desque debe cum plir la for ma ción y or ga niza ción de colecciones, el entorno social en que se desenvuelve la biblioteca y la calidad de los servicios que presta o debe de prestar.

Respecto de los servicios bibliotecarioshabríaque es table ceruna diferen cia en trelos fenómenos relativos a su organización y aquéllos relacionados con su funcionamiento. Los aspectosrelativos al funcionamientodelosserviciosbibliote cariostienen una mayor "visibilidad", puesto que involu cran la na tu ra le za del diá lo go que se presen ta o debe pre sen tar se en tre el usua rioy quien presta el servicio. En cambio, la organizacióndeserviciosbibliotecariosestá directamente vinculado al proceso intelectualde in ter pre ta ción de la formay/o los contenidos temáticos de los documentos, para representarlospormediodecódigoso 
palabras con la finalidad de facilitar su organización;este proceso intelectual se expre sa por me dio de sis te mas de con trol administrativo, deinstrumentosdeaccesoala colección y de la disposición de la colección, y únicamente adquieresentido cuandoeldesarrollode coleccionesestáorientado al cumplimiento de intencionalidadeso finalidadesque do tan a la co lec ción de significados relevantes para los miembros de una comunidad.

Si el proceso organizador de los servicios bibliotecarios exclusivamente se orienta hacia la búsqueda de la eficiencia por medio del refinamiento de las técnicas de administración y margina el hacer explícitas las intenciones, significados y finalidades delaforma ción de colecciones, in du dable mente estaremos frente a la presencia de una maquinaria administrativa que puede ser impresionantemente eficaz para resolver los problemas del control bibliográfico,pero que poco ten drá para ofre cer al individuo que pretendeob te neruno ovarios documentosque contenganlos datos, la información, o conocimientos que requiere, puesto que los instrumentos de control bibliográfico únicamente han contribuido a avasallaral individuoainterminableslistados de información bibliográfica.

Laobsesivapretensión detratarderesolver todas las cuestiones inherentes al control bibliográficopor el fácilyexpeditocamino de la automatización, aplicada en sus aspectosmásprimitivos (capacidaddealmacenamiento y velocidad de recuperación), cuya intencionalidad esencial radica en la construcción de técnicas que sean funciona les para ha cer efi cien te el pro ce so de recuperación de datos, únicamente ha conducido a hacer hincapié en un servicio orientadoaproveer de información bibliográficaalusuario, de manera, que tanto la organización de las colecciones, como los índices y catálogos para el acceso a los documentos se dirigen a la localización de informaciónydatosespecíficos, relegando a un se gun do pla no el es ta ble cery mos trar las relaciones que existen entre diferentes documentos.

Con el desarrollo de las tecnologías denominadas de "inteligencia artificial", se abrieronnuevasalternativasalprocesoautomatizado de registros bibliográficos, dado que este tipo de tecnología hace hincapié en la construcción de "bases de conocimientos" construidasfundamental menteporlas relaciones entre los fenómenos registrados en una base de datos. Desde el punto de vista de la tecnología de inteligencia artificial, el aspecto fundamental del proceso automatizado ya no radica en la búsqueda y recuperación de un dato, sino en la posibilidad que existe de preguntar por las relaciones entre diversosfenómenos, la máquina realiza un proceso de inferencia a partir de re- glas (que expresanlasrelacionesentre dife rentes fenómenos), y busca en la "base de conocimientos" (un archivo con lasparticur laridades de los fenómenos), aquellosfe nó me nos que sean váli dos parala re la ción por la cual se pregunta.

En el ámbitobibliotecológico en la década de los ochenta se intentó aprovechar los sistemas de descripción y clasificación de documentos como fundamentos en la construcción de reglas de sistemas construidos valiéndose de la tecnología de "inteligencia artificial", sin embargo, cada vez es más clara la tendencia a considerar que eluso de este tipo de tec nología re quie re de la creación de sólidos fundamentos teóricos en el terreno de la clasificación en $\mathrm{Bi}$ bliotecología, porque como lo expreso Vickery en 1968: "Un registro de máquina no es simplemente una nueva for ma de almacenar y manejar los tradicionales registros de asien to biblio gráfico, es un me diofísico nuevo y diferente, que tiene usos distintosalosdelosregistrostradicionales" (Vickery (1968), p. 428).

Laúnicarazón para crearregis trosau to mati za dos, es que la má qui na nos ofre ce la po sibilidad de usar los registros bibliográficos de ma ne ras dis tin tas, a comolos he mosvenido utilizando. El manejo automatizado de los datos por medio de tecnologías como la de inteligencia artificial nos abre un mayor, más holgado y flexible proceso de la información y sus relaciones, peroes necesario especificar los propósitos a los que pue de ser vir, y las for mas que pue den uti lizar se en or den a servir a es tos propósi tos.

Larepresentación delconocimientohasido una preocupación constante en el ámbito bibliotecológico, no sóloloquerespectaala construcción de sistemas de clasificación, sino también, porque la práctica bibliotecaria obliga cotidianamente a plantearse el proble madelare presen ta ción de los contenidos documentales, y aun cuando existen gran cantidad de sistemas y teorías sobre el particular, no podemos decir que se haya escrito la última palabra al respecto; por el contrario, la posibilidad de representar el conocimiento mediante símbolos estructuradosen formadere gis tros, programasyarchivospropiosdel terre nodelaau to matización, abre nuevas perspectivas y debe dar lugar a la reflexión acerca de cómo utilizar estas tecnologías como herramientas para imprimirun nuevosen tidoalaforma ción de colecciones, con la intención de ir más allá delsimplecontrolbiliográficoquesedetiene en el almacenamiento-recuperación, y ahon dar más en la crea ción de sis te mas automatizados que nos permitan describir, analizar y relacionar los contenidos temáticos de una colección, con objeto de fundamentaryresignificarlosserviciosbibliotecarios.
La fundamentación de los servicios bibliotecarios evidentemente está relacionada con aspectos inherentes a la lógica de la construcción de sistemas de clasificación y aladeterminación designificadosquesele otorguenalos contenidos de una colección documental.Perosobretodo, implicaestable cerlas cualidadesque debere unir loservicios bibliotecarios para responder a las necesidades de un mundo que induce al uso de la información en forma rápida y pre cisa, pero, al mis motiem po, re quiere de elementosyespaciosparaque el in dividuo pueda generar e integrar conocimientos que le permitan no sólo explicarse lo que suce de en el mundo, sino que con tribuyan a enriquecer su concepción individual del mismo. Aeste res pec to, exis te mu cho por de cir, de ba tir y ha cer, acer ca de lo que la organización de colecciones documentales pue de lo grar para crear nú cleos deconocimienos que ayuden a los individuos a ob te ner una con cep ción más am plia so bre aquellos asuntos para los cuales acude al uso de una colección documental.

\section{LA REPRESENTACION DEL CO- NOCIMIENTO}

Enor mes cam biosen la so cie dad mo derna pueden ser asociados con el concepto de información, este concepto en nuestra sociedad está ligado de varias maneras a los símbolosquemanejandiversoscírculossocialesyalas for mas que uti lizan para lla mar nues traaten ción en distintosmomentos de nuestra vida. Asimismo, las teorías que producenun manejosim bólico delainformación han forzado cambios en distintas disciplinas y han generado apreciaciones culturalesque motivan en bue namedidaa considerar nuestra época como una "era de la in for ma ción", en la cual el pro ce so de "simbolización" como una for ma de re presentación y transmisión de la información tie ne un va lor so cial, y en cier to sen ti dopodríamos hablar de que conforma un lenguaje.

La idea de la posibilidad de representar el conocimiento mediante una combinación de símbolos ha dado lugar a una amplia gama de tecnologías que aprovechan las características de las máquinas computadoras como instrumentos para resolver problemas que pueden expresarse en forma de "fenómenos" y de relaciones entre éstos. La mayor parte de estas tecnologías están sustentadas en la idea de diseñar un lenguaje de máquina computadora que posibilite al programador especificar sus problemas en forma de expresiones lógicas, en vez de las construcciones convencionalesdeprogramacióndirigidasaencadenar instrucciones sobre lo que debe hacer la computadora y en qué momento. Alrededorde 1970 fue inventadoporAlain Colmeuerysuscolaboradoresun nevolenguaje al que denominaron PROgramación 
en LOGica, y que es co no ci do por su abreviación PROLOG, dan dolu garalde sa rrollo de tecnologías como las de "sistemas expertos" y a la "programaciónorientada a objetos".

La programación orientada a objetos, no utiliza algoritmos para estructurar los progra mas de la má qui na, usa objeos comoes tructuras lógicas fundamentales. Estas estructuras se construyen a partir de una abstraccióndelascaracterísticasesenciales de un objeto, distinguiéndolo de todos los de más tipos de obje tos, de esta ma ne raun objetosedelimitaconceptualmenteapartir deparáme tros relativosala perspectivade quien determina la abstracción del objeto. Estasabstraccionesse "jerarquizan", esdecir, se ordenan. Las jerarquizaciones fundamentalmente pueden ser de dos tipos:

a) jerarquización por tipos, que implica la comprensión de un problema mediante la caracterización precisa de las propiedadesestructuralesodecomportamiento comunes a alguna colección de entidades, con lo cual se obtiene una estructura de clases;

b) jerarquización por partes, cuyo objetivo es estructurar los objetos involucrados en el problema.

Cadauno delos obje tos involu cradosenla definicióndeunproblemadeberelacionarse con al gu na de las cla ses. Las cla ses se rela cio nan unas con otras. Los pro gra mas es tán organizados como colecciones de objetos, cada uno de los cuales representa un casoespe cíficodealgu na clase, y cuyas clases pertenecen a una jerarquización de clases unidas por medio de relaciones de herencia.

Este método de programación permite estructurarlainfor ma ción aprocesarporuna máquina en forma de proposiciones lógicas, que pue de adop tar la for ma de pro posiciones linguísticas que establezcan una relación entre dos objetos. Estas proposiciones pueden afirmar, negar, o producir información sobre "algún hecho". El programa debe contener además reglas o funcionessobrelasrela cionesen tre objetos. A partirdeestoselementoslamáquinapuede responderapreguntassobrelasrela ciones entre los objetos estructurados en el programa.

El método de programación orientada a objetos ha generado dos tipos de problemas, a sa ber: uno re la ti vo a las cien cias de la computación, cuyo objetivo es resolver lascuestionesrelacionadascon la creación detecnología quefa cilitela ela bo ración de este tipo de programas; y otro referido direc ta men te con la con cep ción mis ma de lo que significa el conocimiento, la información y las posibilidades que existen de "simbolizarlo" de tal forma que pueda ser procesado por los rudimentarios instrumentos de lógica desarrollados por la tecnología de la computación. Sin embargo, independientemente de la postura que se adopte frente a este tipo de problemas, es necesario considerar las posibilidades que brinda este tipo de tecnología para el desarrollo de los conocimientosde una discipli na, no sólo por lo que pue da sig ni fi car en cuanto a posibilidades de uso de una máquina, sino fundamentalmente porque representa la oportunidad de replantear viejosproblemasdesdeperspectivaslógicasy tecnológicas inéditas.

Unodelosmúltiplesproblemasqueenfrenta la "simbolización" del conocimiento radicaenlasposibilidadesdeubicarelsignificado de un concepto dado en términos de sus relaciones con otros conceptos. La creación de un símbolorequiere de un referente del mundo exterior (un objeto concre to) o imaginario. Unconceptopuedeser representado por un símbolo, como una palabra o un grupode palabras; el símbolo representa indirectamente el objeto referente, sin embargo, como un mismo referente puede poseer diferentes conceptos para diversas personas, o bien el mismo concepto puede ser representado por distintos símbolos, se da lugar a múltiples significados. Este es un viejo problema de la representación documental que puede ser abordadodesdeunaperspectivaendonde eldiálogosignifica tivo conelusuarioseala determinante más importante.

Lautilizacióndelosconceptos, formaslógicas y tecnología generados a partir de la idea de la representación del conocimiento, alretomarse des deelámbitobibliotecológi co pue de dar lu gar a pers pectivasa partirde las cuales re plan tear los proble mas de la catalogación, con la intención de fundamentar servicios bibliotecarios destinados a abrir un especio que permita al público integrar conocimientos al relacionar las formasy/o contenidosdelos diferentesdocumentos que conforman una colección.

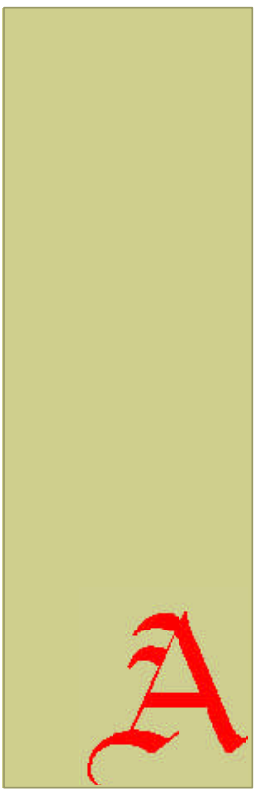

"Uno de los múltiples problemas que enfrenta la 'simbolización' del conocimiento radica en las posibilidades de ubicar el significado de un concepto dado en términos de sus relaciones con otros conceptos"

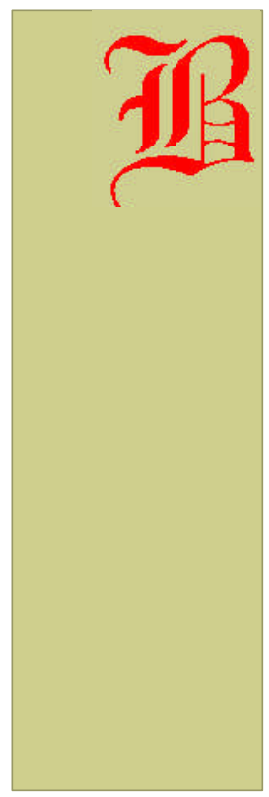


Tratar acerca de la filosofía que debe regir lainvestiga cióny desarrollodeunadisciplina, no se circunscribe a pretender proveer derespuestas a una se rie de interrogantes, oa "proporcionarinformación paralatomade decisiones", según mienten der, estápróxi ma a la idea de in ten tar apren der la na tu raleza de los fenómenos, pero vinculándolos especialmente a lo que pueden significar para la circunstancia humana en determinados momentos y condiciones. Es indispen sable no sólorescatary plan tear problemas, sino colocarlos dentro de una perspectivadistintaalacualordinariamente los contemplamos, con objeto de articularlos en torno a las necesidades humanas para encontrar alternativas de solución, o al menos si ésta no existe, plantear los cami nos para ma ne jar y con vi vir con los proble mas. Es en este sen ti do, que a con ti nuación se presen taun es bozo de lo que po dría ser el contemplar la catalogación como un proble maen don de en tran en jue go "objetos" y "relaciones entre objetos".

\section{LA R E P R E E N T A C I O N DE DOCUMENTOS Y SUS CONTENI- DOS}

Enelámbitobibliotecológicolarepresentación de los documentos y sus contenidos tie ne lain ten ción de ha cer pa ten tes, ante la presen ciadelpúblico, tantolascaracterísti cas físicas y/o contenidos temáticos de un documento, como las re la cio nes que guardan en tresílos diferentes do cumen tosque forman una colección. La representación de documentos puede expresarse de dos formas:

a) por medio de símbolos que representan los contenidos documentales y sirven para acomodar los documentos en un orden predeterminado, $\mathrm{y} / \mathrm{o}$;

b) a través de un registro que contiene la descripción de las características físicas y/o temáticas de un documento.

Tanto los símbolos creados a partir de un sistema de clasificación (Dewey, LC, CDU, $\mathrm{u}$ otros), como los registros realizados en forma de fichas o registros electrónicos, parare presentarun do cumen to (libro, material hemerográfico, revista, video, etc.), muestran las relaciones que se establecen entre diferentesfenómenos (características físicas y/o contenidos temáticos).

Al re pre sen tar cual quier tipo de do cu mento por medio de un registro construido específicamenteparafigurarenlugardeéste, se preten de quelare presenta cióntengalas cualidadesnecesariaspararelacionarcuat quiera de los fenómenos incluidos como par te de un re gis tro, con los fe nó me nos de otro registro. Las relaciones que se establecen entre los diferentes

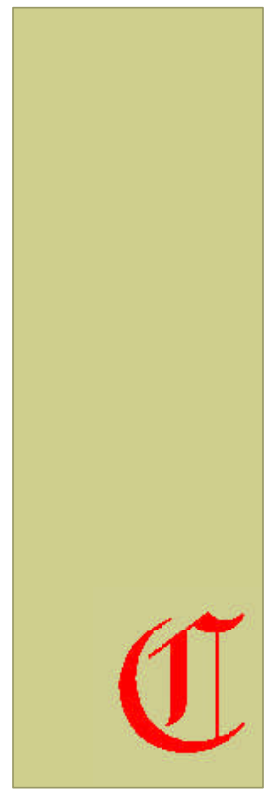

"La representación de los documentos y sus contenidos tiene la intención de hacer

patentes, ante la presencia del público, tanto las características físicas $y / o$ contenidos temáticos de un documento, como las relaciones que guardan entre sí los diferentes documentos que forman una colección"

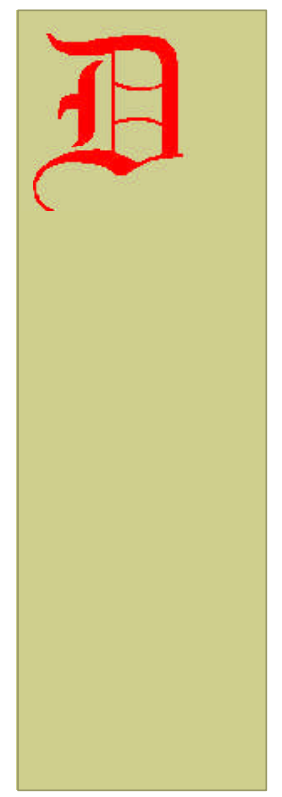

fenómenos que componen los registros, tie nen la fi na li dad de crear un or den do cur mental que hace explícitos los vínculos existentes entre diversos documentos.

Un registro, construido específicamente para representar un documento, lo utilizamos para expresar las relaciones que existen entre fenómenos referidos a un documentosen particular. Por ejem plo, cuando decimos "El libro escrito por García Márquez se ti tu la Cien años de Soledad", es tamos expresando una re la ción en tre un obje to "Unlibro es critopor García Marquez", y otro objeto en particular "ellibrotitulado Cien años de Soledad". Además, la relación tiene un orden específico: es el libro escrito por García Marquez el que tiene el título "Cien años de Sole dad", y no es el título el que tiene un libro.

Algunas re la cio nes que apa re cen en los regis tros para re pre sen taralos do cumen tosy sus contenidos no siempre mencionan todos los fenómenos que entran en juego. Por ejemplo, cuando decimos "El libro titulado ElHom breGramatical es un libro de divulgación científica", queremos decir que existe una relación denominada "divulgación científica", que se aplica al libro "ElHombreGramatical". Sin em bargo, no se men cio na quién con si de ra que esta obra es de divulgación científica ni por qué. $\mathrm{El}$ detallar todos los fenómenos implicados en una re la ción de pen de de qué es lo que se quie re de cir, y de las finali da des que se persigan al representar un documento.

En Bibliotecología es familiar el uso de reglasparadescribirrelacionesen trefenóme nos utilizados para representar un documento. Por ejemplo, la regla que nos dice que "la descripción física de un libro debe con te nerpor lo me nos el au tor, el títuloyel año de pu bli ca ción", nos dice "algo" acerca de lo que significa un libro, a saber: que loescribeunapersonadenominadaautor, que tie ne un nom bre de no minadotítulo, $y$ una fe cha de publica ción. Tam bién no dice comopo de mosave riguaracerca de la exis tencia de un libro: simplemente hay que pre gun tarporsu au tor o el título. Es fun damen tal des ta car que las re glas casi siem pre están demasiado simplificadas, pero que son aceptables como "definiciones". Después de todo no puede esperarse que una definición nos diga todo acerca de algo. Por ejem plo, la ma yo ría de las perso nases taría de acuerdo en que un libro implica mu cho más de lo ex pre sa do por la re gla antes mencionada. No obstante, cuando tratamos de resolver problemas de representacióndocumentalnecesitamosapoyarnos en aque llas re glas que nos ayu dan a re solver el problema.

Siempre debemos tener en consideración el uso de las re glas, a pe sar de su sim pli ficación,por que no sólo ayu dan a los pro pó si- 
tos de resolver los problemas de representación documental, su utilización implica lograr una consistencia en la representación, la cual nospermitere la cio narocompararlosfe nóme nos queutiliza mospararepresentar un documento con aquellos fenómenosqueseutilicenpararepresentar otro do cumen to. Por ejem plo, siusa mosla regla que dice que para describir un libro debemos referirnos al autor, título y fecha; todas las descripciones de libros realizadas conforme a esa regla están relacionadas entre sí en razón de que en todas las descripciones intervienenlosmismosfenómenosylas mis mas relaciones. De otra for ma, siutiliza mosunare gla para des cribirellibro 1 y otra para el li bro 2 , ob ten dre mos dos tipos de descripción que pueden o no relacionarsedependiendo de los fenómenos y rela cionesque in tervengan en cadaunade las descripciones. Si las reglas que se utilizanimpiden onorelacio narlosfenómenos de las descripciones efectuadas con la intención de representar un documento, se perderíaelsentidodelarepresentacióndocumental: establecer relaciones entre distintos documentos.

Laconstrucciónderepresentaciones docut men talestie ne un carácter complejo, es decir, para ex pre sar las re la cio nes que se presentan entre lo diversos fenómenos referidosaundocumento, esindispensable recurrir a distintos sistemas de reglas. Por ejemplo, para representar un documento esnecesarioacudiralossiguientessistemas de reglas:

para la descripción física de los documentos están las reglas para descripción establecidas por los ISBD's (InternationalStandardBookDescrip tion), que es unsiste ma de uso ge neralizado;

para determinar los asientos para la estructuracióndecatálogosexistendi versossiste mas dereglas, algu nosproducidos por organismos internacionales de normalización como la ISO y otros de carácter regional como las reglas angloamericanas de catalogación;

paradescribirloscontenidospodemos optar entre varios sistemas de reglas orientados a la clasificación de los contenidos: encabezamientos de materia, tesauros, descriptores, resúmenes, y otros.

Los sistemas de reglas en el ámbito bibliotecológico tienen una característica en común:suestructuralógicapermitecombinar diferentes sistemas de reglas con el propósito de solucionar problemas específicos.

Los sistemas de reglas en el ámbito bibliotecológico, están constituídosporexpresiones lingüísticas a las que se les denomina reglas. Cada una de las reglas enuncia los fenómenosreferidosadeterminadotipode documentos,yrepresentaestosfenómenos mediante "términos" que con la expresión lingüística de todo lo que pue daser objeto de pensamiento, o darse en cualquier proposición verdadera o falsa. Por ejemplo, el ISBD-G contiene las reglas que expresan cuales son los fenómenos que puedenreferirse para describir los elementos físicos que caracterizan un documento. Una de sus reglas establece que los fenóme nosparala des crip ción de un do cumento deberán de agruparse en las siguientes áreas:

Area de título y de mención de responsabilidad

Area de edición

Area de publicación, distribución, etc.

Area de descripción física

Area de serie

Area de notas

Area de número normalizado

La regla enuncia los fenómenos que pueden estar referidos a la descripción de un documento(autores, editores, nombres de libros, etc.) y los representa mediante una se rie de tér mi nos (Area de títuloy men ción de responsabilidad, Area de edición, Area depublicación,etc.). Además, estableceun orden específico para cada uno de los términos, primerolosagru pa enáreasy de termi na la se cuen cia en la cual se or de nan los términos comprendidos dentro de una Area; dispone también un orden para las áreas, que es el re la tivo a la for ma en que se enumeran las Areas. Por ejemplo,primero aelArea de títuloymen ción deresponsabili dad, lue go el Area de edi ción y así su ce si vamente; dentro de cada área también existe un orden, primerovael títuloylue go la mención de responsabilidad.

El orden y las relacionesentre los términos de un re gis tro do tan de significa doal re gis tro y permite la relación de los contenidos de un re gis tro con los con te ni dos de otro registro. El significado de un registro es consecuencia de la determinación que hacen las reglas acerca de los términos que se considera sirven para describir un documen to, por que és tas enun cian lo que se es tima significativo para representar un documento. Por ejemplo, la aplicación de la regla que establece que el autor y el editor sonfenómenossignificativosparadescribir un documento, daría como consecuencia la creación de varios registros que tienen nombresdeautoresyeditores, los nom bres de autores tienen algo en común: su calidad de autores, lo cual crea una relación entre todos ellos, pero además existe una relación más: la de los autores con los editores.

Al delimitar cuáles son los fenómenos que al referirlos a un documento en particular creanrelacionessignificativas para ese documento, seestableceunaconsistenciaque hace factible la relación entre los contenidos de distintos registros. Un registroaisladoúnicamentenospermitesaberacercade undocumen to. Sonlasrelacionesentrelos contenidos de varios registros las que nos permiten establecer un orden documental que hace factible conocer acerca de una colección de documentos.

La re pre sen ta ción de la for may/o con te ni dos de un documentos nos refiere a sistemas de reglas que determinan los fenómenos y las relaciones que se estiman significativosparadescribirundocumento. Sinembargo, lare presen ta ciónúnicamente nos permiteconocer acerca de un documento; las relaciones que puedan establecerse entre la forma y/o contenidos de variosdocumentosdependedelaclasificación.

En el ámbito bibliotecológico la clasificacióndelimitaununiversodocumental, que en una primera instancia permite determinar si un documento en particular pue deo no incluirse como parte de ese universo, pero además crea un espacio en donde se establecenrelacioneslógicasentrelosfenómenos representados en los registros que describen un documento, relaciones que dotan de significado a cada uno de los fenómenos representados en los registros, significados que sirven de base para la organización física de los documentos y sus representaciones. En otra forma, podríamos decir que la clasificación crea un núcleo de conocimientos, porque al crear relaciones lógicasen treloscontenidos de diversosdocumentos, se obtienen las relaciones entre los conceptos vertidos en los contenidos de los documentos.

El tra bajo de descrip ción de la for may/o contenidostemáticos de un documento presenta varias facetas que se realizan simultáneamente, fundamentalmente radica en la habilidad intelectual para reconocer los contextos y significados de un do cu men to, para en una se gun da instancia determinar la aplicabilidad de una regla específica de algún sistema de descripción y/o clasificación con la intención de ob te nerunare presen ta ción simbólica de los contextos y significados de los contenidos temáticos de un documento. Una tercera instancia la constituye la creación de relaciones entre los contenidos temáticos de los documentos que forman par te de una colección, y que en úl ti ma ins tancia constituirían el conocimiento a simbolizar para construir una base de conocimientos.

Tantolaclasificacióncomolosserviciosbibliote carioshanestadoorientadosalalmacenamien- 
toyrecuperación de la in for ma ción, con la in tro duc ción de la tec no lo gía de los sis temas expertos, se ha mostrado una tenden cia a que la idea de la bús que da de información bibliográfica, no se detenga simplementeenlarecuperación del dato y la información relevante, sino que se mues tran las re la cio nes que existen en tre diversos fenómenos que se encuentran represen ta dos, de tal ma ne ra que no tra taría de in da gar acer ca de un dato en sí, sino acerca de la relación que un fenómeno guarda con otro fenómeno.

Laclasificaciónenelámbitobibliotecológico al delimitar un universo documental y crear un espacio donde se establecen relaciones lógicas entre los fenómenos selec- cionados para representar un documento, con la fi nali dad de crear un or den que hace explícitos los vínculos existentes entre diversos documentos, facilita la organización documental por medio de la formación de colecciones documentales en un lugarespecífico comopue deserunabiblioteca, o un cen tro de in for ma ción, un ar chi vo, entre otros.

\section{REFERENCIAS BIBLIOGRAFICAS}

AGUSTI-CULLELy C.Sierra "Una me to dologíay un len guaje parala in geniería del conocimiento". En Jornadas españolas de documentaciónautomatizada:Documat90 (3: 1990:Palma). Pal ma:Universitat de les Illes Balears, 1990. vol 1. p. 14-27.

AUSTIN, Derek. "Prospects for a new ge ne ral classi fi ca tion" Jour nal of Librarianship, julio 1969. p. 149-169.

BELKIN, Nicholas J. Alina Vickery. Interaction in information systems: A review of research from documentre trieval toknowledge-basedsys tems. Londres, The British Library, 1985. 250p.

BRODIE. Mi chaelL. y Mathias Jarke. "On in te gra ting Lo gic Pro gramming and Databases" EnExpertdatabasesystems:Proceedingsfrom the firts international workshop. editor Larry KeKerschberg. Menlo Park, California: The Benjamin/Cummings, 1986. a. 191-207

CLASSIFICATION Re search Group. "The need for a facetedclassification as the basis of all methods of information retrieval" En Theory of Subjet Analysis: Asource Book/ edited by Louis Mai Chanet. Littleton, Colorado: Libraries Unlimited, 1985. p. 154-167.

COATES, E.J. "Some properties of relationships in the structure of indexing languaje". Progress in Documentation, v. 29, n. 4, b.p. 390304.

GRAIG, I.D. "The Ariadne-1 Blacboard Sys tem" The Com puter Jour nal, v. 29, n. 3, 1986. p. 235-240.

DAHAL, Verónica. "Logic programming for constructive expertdatabase systems". EnExpertda tabase systems: Proceedingsfrom the firts internationalworkshop/editorLa rryKerschberg. MenloPark, California: The Benjamin/Cummings, 1986. p. 209-215.

DAVIS, Roy. "Outlines ofemergingparadigmincataloguing”. Information Proccesing and Management. v. 23, n. 2, p. 89-98

LAFUENTE LOPEZ, Ramiro. Los Sistemas Bibliotecológicos de Clasificación. México, 1991. 200 h. 
LANGRIDGE, D.W. "Disciplines, forms and phenomena". En Ranganathan's Philosophy:Assessment,Impact and Relevance/ edited by T.S. Rajagopalan, Vickas., 1986. p. 222-227.

PAPAKONSTANTINOU, G. y J. Kon tos. "A Knowledge re pre sen ta tion whit attribute nmgrammars"The computerJournal, v. 29, n. 3, 1986. p. 241-245.

RANJANA, Vohra. "Analytico-Syntheticschemeof classificationitsimpact to mo dern theo ries of clas si fication" EnRanganathan'sPhilosophy: Assessment, Impact and Relevance edited by T.S. Rajagopalan, Vickas, 1986. p. 222-227

RIDLEY, M. J. "An expert system for quality control and duplicate detection in biblio gra phic da ta ba ses" Program, v. 26, no. 1, ene ro 1992. p. $1-18$.

SPARK Jones, Karen. "Intelligent interfaces for information retrieval systems:architecture problemsinthe construction of expertsystemsfor documentalretrieval". EnFuturatrendsin informationscienceand technology. P.A. Yates-Mercer, p. 47-73.

VERONA, Eva. "A de ca de of IFLA's work on the stan dar tiza tion of bi bliographic description” IFLA Journal. v. 6, n. 3, 1980. p. 216-233.

VICKERY (1968), B.c. "Bibliographic des cription, arrange mentand retrieval. Journal of documentation. v. 24, n.1 (marzo 1968).

VICKERY, B.C. "Snowledge representation a brief review" The Jornal of documentation. v. 42, n. 3, sep. 86, p. 145-159. 